\title{
Nanotube-Based Controlled Drug Delivery
}

\section{Guofang Chen*}

Department of Chemistry, St. John's University, Queens, NY 11439, USA

Controlled drug delivery systems have already had an enormous impact on medical technology with the potential to improve human health. An important prerequisite for designing an efficient controlled delivery system is the ability to transport the drug molecules with "zero release" to the targeted site and release them in a controlled manner at the targeted site. It will be even more beneficial if the drug molecules are delivered by a system that "senses" or detects the biological stimulus signals caused by disease and responds by releasing the appropriate amount of drug. Many drug delivery systems have been introduced, such as nanoscale drug delivery devices (nanoparticles, nanocapsules, nanotubes, nanogels and dendrimers), porous materials (porous silicon and mesoporous silica) and soft drug delivery systems (micelles, polymer hydrogels, liposomes, microemulsions, capsules) [1-3]. Each system has its advantages and disadvantages. For example, some systems have a high drug loading capacity but their release profiles are more difficult to regulate. Certain delivery systems readily reach specific sites but their drug loading capacity is relatively low.

Nanotubes, hollow cylindrical nanostructures are promising drug carriers offering many advantages over other drug delivery systems. First of all, nanotubes can effectively protect entrapped drug molecules against denaturation or degradation over the delivery process. Further, nanotubes have large inner volumes that can allow for loading more than one therapeutic agent so that targeting molecules, contrast agents, drugs or reporter molecules can be used at the same time. Moreover, nanotubes have open ends which can increase drug loading efficiency. Last, but not the least, nanotubes have separated inner and outer surfaces, which can be differentially functionalized to load desired molecules inside, but impart chemical features to the outer surface allowing for site-specific drug delivery. A variety of organic and inorganic nanotubes have been used to develop effective controlled drug delivery systems. The following nanotube-based drug delivery systems were reported: carbon nanotubes, silica nanotubes, selfassembling lipid nanotubes and polymer nanotubes.

Carbon nanotubes have unique electronic, mechanical, and structural properties as well as chemical stability, which make them ideal nanomaterials for drug delivery carriers. The organic functionalization of carbon nanotubes can improve substantially their solubility and biocompatibility profile with low cytotoxicity so that functionalized carbon nanotubes hold strong promise as novel systems for the delivery of drugs, antigens and genes. A number of reviews have been published outlining the current state of research into targeted drug delivery using carbon nanotubes [4-7].

Silica nanotubes are a novel class of inorganic structures as an ideal vehicle for drug delivery and controlled release because they are easy to make, readily dispersed in aqueous solution and are biocompatible [8]. They can be fabricated by "template synthesis" and differentially functionalized on their inner and outer surfaces. This unique strategy provides monodisperse nanotube dimensions (size and diameter) with features useful as carriers of large amounts of bioactive agents including proteins and genes. Their open ends can also be used as a gate to control drug release. Application of an external stimulus in the form of ultrasound to or chemical functionalization of synthesized silica nanotubes results in higher yield of drug release as well as yield of drug release varying linearly with time [9]. Moreover, two kinds of inorganic/organic hybrid composites based on mesoporous silica nanotubes and $\mathrm{pH}$-responsive polyelectrolytes have been developed as $\mathrm{pH}$-controlled drug delivery systems via the layer by layer self-assembly technique [10]. Magnetic nanotubes have also been synthesized by forming magnetite layers onto the inner surface of pre-prepared silica nanotubes within the pores of an alumina template. Combining the attractive tubular structure with magnetic properties, silica nanotubes can be an ideal multifunctional platform for magnetic resonance image (MRI) guided delivery [11].

Lipid nanotubes with open-ended, hollow cylindrical structures can be formed via noncovalent self-assembly lipid molecules in liquid media. The well designed lipid nanotubular structure with tunable aspect ratios as well as wall thickness, biocompatible membrane surfaces, and easy surface modification, makes them an ideal nanocarrier for drug delivery [12]. Lipid nanotubes have been used for topical delivery of drug into skin. Fluorescent lipid nanotubes were also utilized simultaneously as drug carriers and biomarkers to track and diagnose effectiveness of the treatment [13].

Compared to lipid nanotubes, polymer nanotubes are mechanically stable and can be added with reactive group, which allows for specific targeting or molecular recognition and can be also modulated by stimuli such as $\mathrm{pH}$, temperature, and net charge for the controlled delivery of therapeutic drugs with increased efficacy and optimized doses. Poly(3,4-ethylenedioxythiophene) conducting polymer nanotubes were prepared for precisely controlled dexamethasone drug release [14]. In our research group, functional polymer nanotubes with active epoxy groups, poly(glycidyl methacrylate-co-ethylene glycol dimethacrylate) were synthesized by surface-initiated photopolymerization pattern transfer method in cylindrical alumina nanopores, which can be used as the scaffold to design controlled drug delivery carriers [15] Molecularly imprinted nanotubes were introduced for enanthoselective drug delivery and controlled release [16]. Moreover, soft, water-filled polymer nanotubes were also prepared via self-assembly of amphiphilic ABA-triblock macromonomers in aqueous media [17].

In summary, although the development of nanotube-based controlled drug delivery systems is still in its infancy, the results highlighted here can certainly be considered promising. We anticipate hollow cylindrical nanotubes as novel controlled drug delivery carriers to play an important role to improve the efficacy of available therapeutics.

*Corresponding author: Guofang Chen, Department of Chemistry, St. John's University, Queens, NY 11439, USA, E-mail: cheng@stjohns.edu

Received November 17, 2012; Accepted November 19, 2012; Published November 21, 2012

Citation: Chen G (2012) Nanotube-Based Controlled Drug Delivery. Pharmaceut Anal Acta 3: e136. doi:10.4172/2153-2435.1000e136

Copyright: (c) 2012 Chen G. This is an open-access article distributed under the terms of the Creative Commons Attribution License, which permits unrestricted use, distribution, and reproduction in any medium, provided the original author and source are credited. 


\section{References}

1. Goldberg M, Langer R, Jia X (2007) Nanostructured materials for applications in drug delivery and tissue engineering. J Biomater Sci Polym Ed 18: 241-268.

2. Anglin EJ, Cheng L, Freeman WR, Sailor MJ (2008) Porous silicon in drug delivery devices and materials. Adv Drug Deliv Rev 60: 1266-1277.

3. Malmsten M (2006) Soft drug delivery systems. Soft Matter 2: 760-769.

4. Bianco A, Kostarelos K, Partidos CD, Prato M (2005) Biomedical applications of functionalized carbon nanotubes. Chem Commun 5: 571-577.

5. Prato M, Kostarelos K, Bianco A (2008) Functionalized Carbon Nanotubes in Drug Design and Discovery. Acc Chem Res 41: 60-68.

6. Yang W, Thordarson P, Gooding JJ, Ringer SP, Braet F (2007) Carbon nanotubes for biological and biomedical applications. Nanotechnology 18.

7. Hilder TA, Hill JM (2009) Modeling the loading and unloading of drugs into nanotubes. Small 5: 300-308.

8. Son SJ, Bai X, Lee SB (2007) Inorganic hollow nanoparticles and nanotubes in nanomedicine. Part 1. Drug/gene delivery applications. Drug Discovery Today 12: $650-656$

9. Kapoor S, Bhattacharyya AJ (2009) Ultrasound-Triggered Controlled Drug Delivery and Biosensing Using Silica Nanotubes. J Phys Chem C 113: 71557163.
10. Yang YJ, Tao X, Hou Q, Ma Y, Chen XL, et al. (2010) Mesoporous silica nanotubes coated with multilayered polyelectrolytes for $\mathrm{pH}$-controlled drug release. Acta Biomaterialia 6: 3092-3100.

11. Son SJ, Reichel J, He B, Schuchman M, Lee SB (2005) Magnetic nanotubes for magnetic-field-assisted bioseparation, biointeraction, and drug delivery. $J$ Am Chem Soc 127: 7316-7317.

12. Hafez IM, Cullis PR (2001) Roles of lipid polymorphism in intracellular delivery. Adv Drug Deliv Rev 47: 139-148.

13. Zhou Y, Kogiso M, He C, Shimizu Y, Koshizaki N, et al. (2007) Fluorescent nanotubes consisting of CdS-Embedded bilayer membranes of a peptide lipid. Advanced Materials 19: 1055-1058

14. Abidian MR, Kim DH, Martin DC (2006) Conducting-polymer nanotubes for controlled drug release. Advanced Materials 18: 405-409.

15. Zou C, Luo Z, Le DH, Dessources K, Robles A, Chen G (2011) Synthesis of polymer nanograss and nanotubes by surface-initiated photopolymerization in cylindrical alumina nanopores. Journal of Materials Chemistry 21: 1454314548.

16. Yin J, Cui $Y$, Yang G, Wang $H$ (2010) Molecularly imprinted nanotubes fo enantioselective drug delivery and controlled release. Chem Commun 41: 7688-7690.

17. Grumelard J, Taubert A, Meier W (2004) Soft nanotubes from amphiphilic ABA triblock macromonomers. Chem Commun 13: 1462-1463. 